\title{
Rapid formation of single-cell pairs for producing hybridomas
}

\author{
Tomoyuki Yasukawa, Masato Suzuki, Fumio Mizutani \\ Graduate School of Material Science, University of Hyogo, 3-2-1, Kouto Kamigori, Ako, Hyogo 678- \\ 1297, Japan
}

\begin{abstract}
IMCS2018_Headline"):
The attractive force of positive dielectrophoresis has been used to form the pairs of different types of cells rapidly and simply. Two different types of cells were successively directed into the individual wells fabricated on the microwell array electrodes. The width $(14 \mu \mathrm{m})$ and depth $(25 \mu \mathrm{m})$ of the microwells restricted the size to two vertically aligned cells. Mouse myeloma cells stained in green were trapped within $1 \mathrm{~s}$ in the microwells by p-DEP by applying an alternating current voltage. The cells were retained inside the wells even after switching off the voltage and washing with a fluidic flow. Cells stained in blue were then trapped in the microwells occupied by the myeloma cells to form the vertical cell pairing in the microwells. Two types of cells were paired within only 1 min and a pairing efficiency of $60 \%$ was achieved.
\end{abstract}

Key words: dielectrophoresis, cell pairs, microwell array electrode, cell fusion, electrofusion, hybrid cells, electroporation

\section{Introduction}

Particles placed in a spatially inhomogeneous electric field experience dielectrophoresis (DEP) force by the interaction of a polarization effect induced in the particles. DEP is attractive for the manipulation of micro- and nano-objects including biological living cells and bacteria and has been used in the wide range of applications, such as a separation and sorting [1], trapping [2] and patterning [3]. Cell arrays can be fabricated using positive DEP ( $p$-DEP), which is a force directed particles towards the regions of electric field maxima $(10,11)$. Generally, p-DEP patterning is utilized to attract in array formats with the pair of electrodes at every elements modified with cell adhesive layers to produce cell patterns.

We used p-DEP to capture cells to microwell arrays fabricated on the ITO substrate and form the vertical alignment of different types of cells. To produce hybrid cells by cell fusion, it is important to form cell pairs with two different types of cells and apply the electric fields parallel to the cell pairs. Thus, vertically aligned pairs of the different types of cells were formed in microwells by $p$-DEP and pairs of cells were fused together by applying electric pulses.

\section{Experimental}

The dielectrophoretic patterning device consisted of an ITO electrode and an ITO microwell array electrode. The number of microwells (width $14 \mu \mathrm{m}$ and depth $16 \mu \mathrm{m}$ ) was set at $250,000(500 \times 500)$. A $30-\mu m$ thick polyester film was used as a spacer to fabricate the fluidic channel between the upper ITO electrode and the lower ITO electrode with the microwell array. Fig. 1 shows an illustration of the formation of pairs of the different types of cells with vertical orientation and the fusion of cell pairs formed in the microwells by electric field pulses. A suspension of myeloma cells stained in green was introduced into the fluidic channel (Fig. 1A). An AC voltage (10 Vpp) in the $\mathrm{p}$-DEP frequency region $(1.0 \mathrm{MHz}$ ) was applied between the upper and lower ITO electrodes. A strong electric field was formed in the microwells to direct cells to the microwells.

A suspension of cells stained in blue with Hoechst 33342 was introduced into the channel (Fig. 1B). The p-DEP was again used to navigate the cells into the microwells with the cells by the first trapping step. Fluorescence images were obtained of the array of pairs of cells stained in two different colors in the microwells. The efficiency of the cell occupation in the microwells was defined as the ratio of the average number of microwells with cells to the 
total number of microwells. Finally, cell pairs formed in the microwells were fused by electric field pulses.
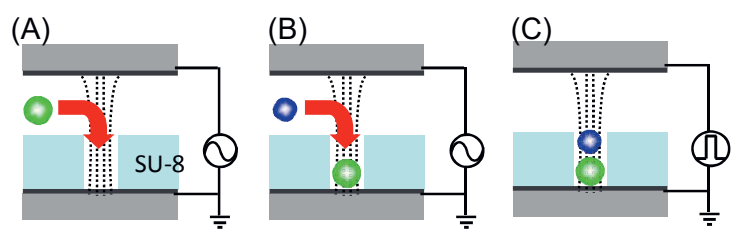

Microwell
electrode

Fig. 1. (A) and (B) Formation of pairs of the different types of cells with vertical orientation and (C) fusion of cell pairs formed in the microwells by electric field pulses.

\section{Results and discussion}

Fig. 2 shows the fabricated microwell array electrode and myeloma cells stained in green in the channel. The suspension of cells was introduced into the channel on the microwell array electrode and an AC voltage of $10 \mathrm{~V}_{\mathrm{pp}}$ was applied at a frequency of $1.0 \mathrm{MHz}$. When suspensions were injected into the channel, cells were randomly dispersed in the channel and were carried by the fluidic flow (Fig. 2B). On applying the $A C$ voltage, the dispersed cells immediately, i.e., within $1 \mathrm{~s}$, stayed at positions with microwells (Fig. 2C). Fluorescence signals were observed at identical intervals after flushing by injecting the sucrose solution (Fig. 2D). The results suggest that stained cells were trapped in microwells (efficiency $80 \%$ ).
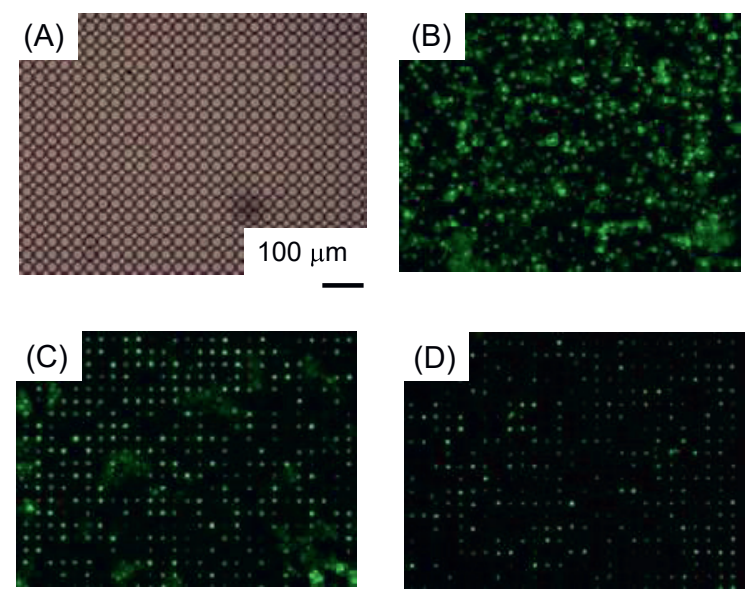

Fig. 2. (A) Fabricated microwell array electrode and myeloma cells stained in green in the channel $(\mathrm{B})$ before and $(\mathrm{C})$ after applying $A C$ voltage and (D) after flushing by injecting the sucrose solution.

No cells was trapped in microwells without the p-DEP attractive force in this experimental period. The removal step of the excess cells of $20 \mathrm{~s}$ was required after the cell trapping for $10 \mathrm{~s}$. Therefore, the single cell array can be efficiently obtained by p-DEP within $30 \mathrm{~s}$. In addition, fluorescence signals were not observed from the positions between the microwells. These results indicate that almost all of the excess cells were removed from the channel after flushing with the fluidic flow.

We performed vertical cell pairing with two different types of cells (myeloma cells and K562 cells) using the p-DEP device. Myeloma cells stained in green were guided to the microwells, then K562 cells stained in blue were successively directed to microwells occupied with myeloma cells. Figs $3 \mathrm{~A}$ and $3 \mathrm{~B}$ show the images of myeloma cells and K562 cells paired in microwells, respectively.

Images were obtained after the removal of excess cells to downstream by injecting a sucrose solution. During cell removal, the $p$ DEP attractive force was applied in order to retain K562 cells positioned on the top component in each pair. Both blue and green signals were observed in individual microwells. Combination image of Figs $3 \mathrm{~A}$ and $3 \mathrm{~B}$ and an optical image of the paired cells are shown in Figs $3 C$ and $3 D$, respectively. Both blue and green signals are clearly observed from the microwells. The observation of the light blue color provides strong evidence for vertical alignment of blue myeloma cells and green K562 cells in the microwell to form single-cell pairs. In this experiment, the cell occupation efficiencies in the microwells were $87 \%$ and $88 \%$ for blue and green cells, respectively. The different types of cells can be vertically paired within only $1 \mathrm{~min}$ with a pairing efficiency of $53 \%$.
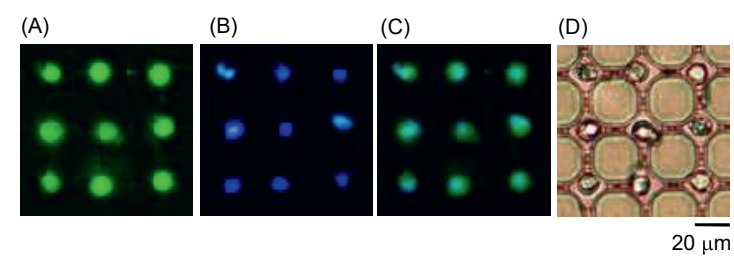

Fig. 3. Images of (A) myeloma cells and (B) K562 cells paired in microwells. (C) Combination image of Figs $3 A$ and $3 B$ and (D) optical image of the paired cells.

\section{References}

[1] T. Yasukawa, M. Suzuki, T. Sekiya, H. Shiku, and M. Matsue, Biosens. Bioelectron., 22, 2730-2736 (2007); doi: 10.1016/j.bios.2006.11.010

[2] Y. Yoshimura, M. Tomita, F. Mizutani, T. Yasukawa, Anal. Chem., 86, 6818-6822 (2014); doi: 10.1021/ac5015996

[3] H. Hatanaka, T. Yasukawa, and F. Mizutani, Anal. Chem., 83, 7207-7212 (2011); doi: $10.1021 / a c 201789 m$ 